\title{
An Open Distributed VPN Management System for a Multi- Domain Management World
}

\author{
H. Khayat, L. H. Bjerring, R. S. Lund \\ L. M. Ericsson A/S, Sluseholmen 8, DK-1790 Copenhagen, \\ Denmark
}

\begin{abstract}
Virtual Private Network (VPN) services aim at providing users a single management interface to a heterogeneous global network infrastructure. Various interoperability problems have to be overcome in order to implement this service in a way that it appears to be simple and easy to use. These interoperability problems occur because the global network infrastructure has many owners (expressed as administrative heterogeneity), is constructed by many network technologies (expressed as technological heterogeneity), and because different portions of the network support different management services and modelling representations (expressed as service heterogeneity).

In the ACTS project Prospect a VPN architecture has been defined for managing such a network infrastructure. The base principles underlying the architecture are: (i) to keep things simple, and (ii) to clearly separate between service core and service adaptation functions. This leads to a layered management architecture where the upper layer is responsible for implementing the service logic (mainly concerned with domain level routing), the lowest layer is concerned with adapting heterogeneous management technologies (SNMP, TMN, CORBA) to the technology chosen for implementing the VPN (CORBA), and the middle layer is responsible for adapting the management models associated with each network technology domain to a common, technology-independent abstraction useful for the VPN service logic. The abstraction follows principle (i) of being simple, just as the model applied at the user interface is simple.

A VPN service has been implemented to provide end-to-end connection management support over a heterogeneous pan-European network, providing support for an integrated tele-eduaction service developed by Prospect. The network consists of a set of customer premises networks (ethernet and ATM LAN technologies) managed by SNMP-based management systems, interconnected by a public ATM network managed by a TMN-based management system.
\end{abstract}

Keywords

Virtual Private Network, Inter-Domain Management, End-to-End Management

A. A. Lazar et al. (eds.), Integrated Network Management $V$

(C) Springer Science+Business Media Dordrecht 1997 